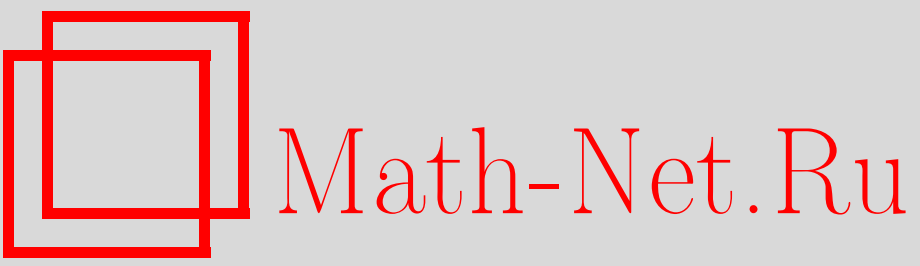

В. Г. Дубровский, Об одном точном решении управляющих уравнений модели обратимого роста, ТМФ, 1996, том 108, номер 2, 327-336

DOI: https://doi.org/10.4213/tmf1194

Использование Общероссийского математического портала Math-Net.Ru подразумевает, что вы прочитали и согласны с пользовательским соглашением

http://www.mathnet.ru/rus/agreement

Параметры загрузки:

IP: 54.89 .56 .158

26 апреля 2023 г., 06:52:24 


\section{В.Г. Дубровский \\ ОБ ОДНОМ ТОЧНОМ РЕШЕНИИ УПРАВЛЯЮЩИХ УРАВНЕНИЙ МОДЕЛИ ОБРАТИМОГО РОСТА}

Проведено исследование системы управляющих уравнений мономерной квазихимической модели обратимого роста в гетерогенной материально открытой среде. Получено точное решение управляющих уравнений в случае линейных по числу частиц констант скоростей реакций роста и распада. Показано, что рассматриваемая модель обладает свойством канонической инвариантности относительно распределения Пойа с изменяющимся во времени средним размером комплексов.

\section{ВВЕДЕНИЕ}

В настоящей работе рассматривается система управляюших уравнений, описывающая гетерогенную среду с компонентами сорта $A$ и $B$, которые реагируют друг с другом по схеме квазихимической мономерной модели обратимого роста $A_{1}+B \rightleftharpoons A_{1} B$; $A_{1} B+A_{1} \rightleftharpoons A_{2} B ; \ldots ; A_{i} B+A_{1} \rightleftharpoons A_{i+1} B \ldots$ Данная модель широко применяется в теориях конденсации в газовой фазе $[1,2]$, начального этапа нуклеации тонких пленок на локальных адсорбционных центрах [3], релаксации колебательно-неравновесного газа [4-7], гетерогенного кластерообразования в гиперзвуковых струях [8], а также для ряда задач биологии и медицины [3]. В отличие от моделей необратимого роста, основанных на различных вариантах уравнения Смолуховского для гомогенной среды с реакциями типа $A_{i}+A_{j} \rightarrow A_{i+j}$ [9], рассматриваемая модель предполагает установление равновесного состояния с конечным средним размером при $t \rightarrow \infty$. Ограничиваясь учетом реакций комплексов $A_{i} B$ только с мономерами, мы считаем концентрацию ядер $B$ достаточно малой, чтобы можно было пренебречь межкластерными процессами $A_{i} B+A_{j} B \rightleftharpoons A_{i+j} B$ [10]. В такой модели вьполняется условие нормировки, или сохранение нулевого момента функции распределения по размерам, а первый момент равен среднему размеру. Это отличает рассматриваемую ситуацию от случая гомогенной среды, где полное число комплексов не сохраняется, а средний размер дается вторым моментом распределения [9].

Хорошо разработаны различные приближенные методы решения систем управляющих уравнений со сложной зависимостью констант скоростей от размера, такие как континуальное приближение $[1,11]$, методы теории возмушений $[4,12]$, релаксационные представления $[2,7]$. Известно небольшое число точно решаемых случаев в различных моделях реакций, которые обычно связаны с выбором упрошенных констант скоростей. Так, точные решения уравнения Смолуховского для произвольных начальных распределений известны при коагуляционных ядрах $k_{i j}=$ const; $i+j[13] ; i j$ [14]. Обзор час- 
тично решаемых случаев (например, при монодисперсном начальном условии) можно найти в [9]. Точное решение мономерной модели при постоянных константах скоростей (динамика одномерной модели Изинга с корреляциями между соседними спинами) получено в [15]. Для случая релаксации газа гармонических осцилляторов в приближении одноквантовых переходов точные решения управляющих уравнений даны в $[5,6]$.

Данная работа посвящена построению точного решения управляющих уравнений для системы $A_{i} B+A_{1} \rightleftharpoons A_{i+1} B$ в случае линейных по размеру $i$ констант скоростей. Исследование проводится для материально открытой системы. Анализируются свойства полученного решения, в частности каноническая инвариантность, а также различные частные случаи. Вопросы интерпретации полученных результатов в работе не рассматриваются. Это будет предметом отдельного сообщения. Оказывается, что линейная по числу частиц модель констант скоростей находит применение в биологии и вирусологии (процесс размножения, динамика популяций, распространение вирусных заболеваний).

\section{1. СИСТЕМА УПРАВЛЯЮЩИХ УРАВНЕНИЙ}

Полагая $A_{0} B \equiv B$, квазихимическую схему мономерного обратимого роста можно представить в виде

$$
A_{i} B+A_{1} \rightleftharpoons A_{i+1} B, \quad i=0,1,2, \ldots
$$

Частишы $A_{1}$ будем называть мономерами, частишы $B$ - ядрами, о частище $A_{i} B$, состоящей из $i$ мономеров и одного ядра, будем говорить как о комплексе размера $i$. Прямую и обратные реакции (1) будем трактовать как реакции роста и дробления (распада) комплекса, соответствуюшие константы скоростей обозначим $k_{i}^{+}$и $k_{i+1}^{-}$. Их предполагаем зависящими только от размера $i$. Способность среды к материальному контакту с окружением будем характеризовать положительным и отрицательным источниками накачки мономеров интенсивности $P(t)$ и $Q(t)$, соответственно.

Исходя из соображений баланса для нормированной функции распределения комплексов по размерам $f_{i}(t), i=0,1,2, \ldots$ (при $i=0 \quad f_{0}(t)$ есть доля свободных ядер $B$ ) и концентрации мономеров $f_{A}(t)$ можно записать систему управляюших уравнений [2]

$$
\begin{aligned}
d_{t} f_{A}(t) & =-\sum_{i=1}^{\infty} J_{i}(t)+P(t)-Q(t) f_{A}(t) \\
d_{t} f_{i}(t) & =J_{i}(t)\left(1-\delta_{i, 0}\right)-J_{i+1}(t), \quad i=0,1,2, \ldots, \\
J_{i}(t) & \equiv k_{i-1}^{+} f_{A}(t) f_{i-1}(t)-k_{i}^{-} f_{i}(t), \quad i=1,2,3, \ldots
\end{aligned}
$$

Величина $J_{i}(t)$ имеет смысл тока по оси размеров. Символ Кронекера $\delta_{i, 0}$ в $(3)$ означает запрет на распад ядра $B$. Уравнение (2) отражает факт изменения концентрации мономеров в результате каждой из реакций (1) и за счет источников.

Введем моменты функции распределения порядка $g$ согласно определению

$$
M_{g}(t) \equiv \sum_{i=0}^{\infty} i^{g} f_{i}(t), \quad g=0,1,2, \ldots
$$

Очевидно, первый момент $M_{1}(t)$ есть средний размер комплексов:

$$
M_{1}(t)=\langle i\rangle_{t}
$$


Функция

$$
D(t)=\left\langle\left(i-\langle i\rangle_{t}\right)^{2}\right\rangle_{t}=M_{2}(t)-M_{1}^{2}(t)
$$

есть дисперсия распределения в момент времени $t$. Для нулевого и первого моментов функции распределения из (2), (3) следуют условия нормировки (закон сохранения полного числа ядер) и баланса полного числа мономеров

$$
\begin{aligned}
M_{0}(t) & =1 \\
d_{t} M_{1}(t)+d_{t} f_{A}(t) & =P(t)-Q(t) f_{A}(t) .
\end{aligned}
$$

В латерально изолированной системе $(P=Q=0)$ вместо (9) справедлив закон сохранения полного числа мономеров

$$
M_{1}(t)+f_{A}(t)=\Gamma
$$

где Г есть отношение полного числа частиц сорта $A$ к полному числу ядер $B$.

Система (2)-(4) представляет собой бесконечную цепочку связанных уравнений, наличие интегральных условий баланса делает ее к тому же нелинейной. Аналитические исследования (2)-(4) при сложной зависимости констант скоростей от размера проводятся поэтому в рамках различных приближений. Наиболее распространенным является континуальное приближение, особенно в применении к кинетике гетерогенной нуклеации в газах $[1,2]$ и на поверхности [3]. При этом в свободномолекулярном режиме роста для больших кластеров $k_{i}^{+}=$const $i^{1-1 / d}$, где $d$ - размерность пространства, а $k_{i}^{-}$можно восстановить из $k_{i}^{+}$на основе принципа детального баланса с макроскопической аппроксимацией для свободной энергии образования зародыша. Цепочка уравнений (3) при $i \gg 1$ заменяется одним уравнением в частных производных фоккер-планковского типа, а условия баланса (8), (9) или (10) преобразуются в интегральные уравнения, содержашие долю свободных ядер и пересыщение пара мономеров [1]. Ясно, что континуальное приближение неприменимо в области малых размеров, например для исследования кластерообразования в недосышенных или слабо метастабильных средах [7]. Кроме того, переход к континуальному приближению неоправдан и при очень высоких степенях метастабильности среды $[11,16]$.

Другим часто применяемым методом решения (2)-(4) служит метод приближенной диагонализации трех диагональной матрицы переходов. Один из вариантов диагонализуюшего преобразования был предложен в [7] для случая колебательной релаксации слабоангармонических осцилляторов, а затем применен в [2] для описания гетерогенной конденсации в ламинарном потоке парогазовой смеси. Однако данный подход применим в основном при исследовании состояний, близких к стационарным, и не описывает движение ростового фронта по оси размеров.

Исходя из сказанного ясно, что представляет интерес отыскание моделей, в которых система управляющих уравнений решается точно. Результаты, полученные ниже, обобщают известные решения $[5,6]$ на случай произвольного коэффициента при линейном члене в константе скорости роста, зависимости концентрации мономеров от времени и наличия источников. 


\section{2. РАВНОВЕСНОЕ СОСТОЯНИЕ}

Решение (2)-(4) при $t \rightarrow \infty$ находится из принципа детального баланса $J_{i}(\infty)=0$, $i=1,2,3, \ldots$ Из (4) с учетом (8) для равновесных концентраций $f_{i}(\infty)$ следует известное выражение [2]

$$
\begin{gathered}
f_{i}(\infty)=H^{-1}(x) \zeta_{i} x^{i}, \quad i=1,2,3, \ldots, \\
\zeta_{i} \equiv \prod_{j=0}^{i-1} k_{j}^{+} / k_{j+1}^{-}, \\
H(x) \equiv \sum_{i=0}^{\infty} \zeta_{i} x^{i} \quad\left(\zeta_{0} \equiv 1\right) .
\end{gathered}
$$

Здесь $x \equiv f_{A}(\infty)$ есть равновесная концентрация мономеров. В случае с источниками накачки из $(9),(2)$ заключаем, что равновесие может достигаться только при

$$
f_{A}(\infty)=P(\infty) / Q(\infty) .
$$

В материально изолированной системе для нахождения $f_{A}(\infty)$ используется закон сохранения (10), который при $t \rightarrow \infty$ можно записать в виде

$$
x\left(1+\frac{d \ln H(x)}{d x}\right)=\Gamma .
$$

Из (12) следует, что равновесное распределение зависит лишь от отношения констант скоростей реакций роста и дробления друг к другу $k_{i}=k_{i}^{+} / k_{i+1}^{-}$(термодинамическая константа равновесия реакций (1)). Поэтому равновесное распределение не меняется при переходе от $k_{i}^{ \pm}$к более обшей модели

$$
\tilde{k}_{i}^{+}=k_{i}^{+} \Psi(i), \quad \tilde{k}_{i}^{-}=k_{i}^{-} \Psi(i-1),
$$

где $\Psi(i)$ - произвольная функция размера.

В дальнейшем мы будем интересоваться линейной по размеру комплексов моделью констант скоростей

$$
k_{i}^{+}=k^{+}(1+\alpha i), \quad k_{i}^{-}=k^{-} i, \quad i=0,1,2, \ldots \quad(\alpha>0) .
$$

Коэффициенты $\zeta_{i}$ при этом имеют вид

$$
\zeta_{i}=\left(k^{+} / k^{-}\right)^{i} \prod_{j=0}^{i-1}(1+j \alpha) / i !
$$

Функцию $H(x)$ с коэффициентами (18) легко вычислить, используя равенство

$$
\sum_{i=0}^{\infty} \frac{\prod_{j=0}^{i-1}(1+j \alpha)}{i !} Y^{i}=(1-\alpha Y)^{-1 / \alpha}, \quad \alpha Y<1,
$$


которое проверяется непосредственно разложением правой части в ряд по $Y$ :

$$
H(x)=\left(1-\alpha k^{+} x / k^{-}\right)^{-1 / \alpha}, \quad \alpha k^{+} x / k^{-}<1 .
$$

Подставляя (18), (20) в (11), заключаем, что равновесное состояние в системе с линейными по $i$ константами скоростей, равно как и для более общего случая (16), описывается распределением Пойа

$$
f_{i}(\infty)=\left[1+\alpha M_{1}(\infty)\right]^{-1 / \alpha} \frac{\prod_{j=0}^{i-1}(1+j \alpha)}{i !}\left[\frac{M_{1}(\infty)}{1+\alpha M_{1}(\infty)}\right]^{i} .
$$

Средний размер комплексов при $t \rightarrow \infty$ связан с равновесной концентрацией мономеров соотношением

$$
M_{1}(\infty)=\frac{k^{+} f_{A}(\infty)}{k^{-}-\alpha k^{+} f_{A}(\infty)}
$$

В материально открытой системе $f_{A}(\infty)$ определяется выражением $(14)$, поэтому относительно источников необходимо дополнительно предположить

$$
P(\infty) / Q(\infty)<\frac{k^{-}}{\alpha k^{+}}
$$

Когда неравенство (23) обрашается в равенство, $M_{1}(\infty)=\infty$. Это означает, что рост становится необратимым [9]. Расходимость числа частиц вызвана либо сильной накачкой, либо доминирующими при больших $i$ процессами роста $(\alpha P(\infty) / Q(\infty) \gg 1$ при сравнимых $k^{-}$и $\left.k^{+}\right)$. В обоих случаях система управляюших уравнений (2)-(4) для достаточно больших $t>t_{c}$ становится несправедливой, поскольку при увеличении плотности среды необходим учет межкластерных взаимодействий.

Для материально изолированной системы из (15), (20) нетрудно получить

$$
f_{A}(\infty)=\frac{1}{2}\left(\Gamma+\frac{k^{+}+k^{-}}{\alpha k^{+}}\right)-\frac{1}{2}\left[\left(\Gamma+\frac{k^{+}+k^{-}}{\alpha k^{+}}\right)^{2}-4 \frac{k^{-} \Gamma}{\alpha k^{+}}\right]^{1 / 2}
$$

В данном случае равновесный средний размер положителен и конечен для любых значений $k^{+}, \alpha$ и $k^{-}>0$.

\section{3. ЭВОЛЮЦИЯ ИЗ ПРОИЗВОЛЬНОГО НАЧАЛЬНОГО РАСПРЕДЕЛЕНИЯ}

Обратимся к изучению эволюции системы в равновесное состояние из заданного начального распределения

$$
f_{A}(t=0)=f_{A}(0), \quad f_{i}(t=0)=f_{i}(0) .
$$

Решение управляюших уравнений проше всего получить, воспользовавшись методом производящей функции [6]

$$
f(z, t)=\sum_{i=0}^{\infty} f_{i}(t) z^{i}
$$


Из определения $f(z, t)$ следует

$$
\begin{gathered}
f(1, t)=M_{0}(t)=1, \\
\left.\partial_{z} f(z, t)\right|_{z=1}=M_{1}(t) .
\end{gathered}
$$

Дифференцируя $f(z, t)$ по $t$ и используя (3), (27), в случае констант скоростей (17) получим замкнутое уравнение первого порядка для производяшей функции с начальным условием в виде степенного ряда

$$
\begin{gathered}
\partial_{t} f(z, t)-(z-1)\left[k^{-}-\alpha k^{+} f_{a}(t) z\right] \partial_{z} f(z, t)=(z-1) k^{+} f_{A}(t) f(z, t), \\
f(z, t=0)=\sum_{i=0}^{\infty} f_{i}(0) z^{i}
\end{gathered}
$$

Дифференцируя (29) по $z$ и полагая затем $z=1$, приходим к уравнению для среднего размера

$$
d_{t} M_{1}(t)+\left(k^{-}-\alpha k^{+} f_{A}(t)\right) M_{1}(t)=k^{+} f_{A}(t)
$$

Совместно с условием баланса (9) это дает систему уравнений для определения $M_{1}(t)$ и $f_{A}(t)$ в материально открытой системе при заданных источниках $P(t), Q(t)$. Ее можно свести к одному уравнению второго порядка для среднего размера

$$
\begin{gathered}
\left(1+\alpha M_{1}\right)^{-1} \ddot{M}_{1}-\alpha\left(1+\alpha M_{1}\right)^{-2} \dot{M}_{1}^{2}+ \\
+\left[k^{+}+\left(1+\alpha M_{1}\right)^{-2} k^{-}+\left(1+\alpha M_{1}\right)^{-1} Q\right] \dot{M}_{1}+ \\
+\left(1+\alpha M_{1}\right)^{-1} k^{-} Q M_{1}=k^{+} P \\
M_{1}(t=0)=M_{1}(0) \\
\dot{M}_{1}(t=0)=-\alpha k^{+} M_{1}^{2}(0)+\left[\alpha k^{+} \Gamma(0)-k^{+}-k^{-}\right] M_{1}(0)+k^{+} \Gamma(0),
\end{gathered}
$$

где $\Gamma(0)=M_{1}(0)+f_{A}(0)$ есть отношение полных концентраций частиц $A$ и $B$ при $t=0$.

В материально изолированной системе уравнение (31) замкнуто относительно $M_{1}(t)$, его решение будет приведено ниже. Если считать $f_{A}(t)$ известной функцией, формальное решение (31) дает связь между $M_{1}(t)$ и $f_{A}(t)$, которую можно представить в виде

$$
\begin{gathered}
M_{1}(t)=G(t)+M_{1}(0) \exp (-F(t)), \\
F(t)=k^{-} t-\alpha k^{+} \int_{0}^{t} d t^{\prime} f_{A}\left(t^{\prime}\right), \\
G(t)=\exp (-F(t)) \int_{0}^{t} d t^{\prime} f_{A}\left(t^{\prime}\right) \exp \left(F\left(t^{\prime}\right)\right) .
\end{gathered}
$$

Очевидно, $G(t)$ есть средний размер в момент времени $t$ при условии $M_{1}(0)=0$. Из (34), (35) следует, что равновесное состояние с конечным средним размером достигается независимо от начальных условий при $\lim _{t \rightarrow \infty} F(t)=\infty$, или $k^{-}-\alpha k^{+} f_{A}(\infty)>0$. Как мы видели, это условие всегда выполнено для изолированных систем, а также для открытых систем при $P(\infty)$ и $Q(\infty)$, удовлетворяющих $(23)$. Концентрация мономеров восстанавливается из решения (32), (33) с помошью (31):

$$
f_{A}(t)=\left(d_{t} M_{1}(t)+k^{-} M_{1}(t)\right) / k^{+}\left(1+\alpha M_{1}(t)\right),
$$


что при $t \rightarrow \infty$ совпадает с (22).

При вычислении первых интегралов (29) возникает уравнение Риккати специального вида, имеюшее частное решение $z=1$. Это обстоятельство позволяет получить решение (29) с начальным условием (30) в явном виде

$$
f(z, t)=[1+\alpha G(t)(1-z)]^{-1 / \alpha} \Sigma
$$

где

$$
\Sigma=\sum_{i=0}^{\infty} f_{i}(0)\left[\frac{1+(\alpha G(t)-\exp (-F(t)))(1-z)}{1+\alpha G(t)(1-z)}\right]^{i} .
$$

Легко убедиться, что (38) обладает свойствами $(27),(28)$. Поскольку $G(t)=M_{1}(t)$ при $M_{1}(0)=0$ и $\exp (-F(t))=\left(M_{1}(t)-G(t)\right) / M_{1}(0)$ для $M_{1}(0)>0$, для окончательного определения производящей функции, а с ней и распределения комплексов по размерам требуется лишш решение уравнения (31) или (33). При $t \rightarrow \infty M_{1}(\infty)=G(\infty)$, поэтому

$$
f(z, \infty)=\left[1+\alpha M_{1}(\infty)(1-z)\right]^{-1 / \alpha}
$$

Коэффициенты разложения (39) в ряд по z дают равновесное распределение Пойа (21).

Таким образом, (38) есть точное решение динамической задачи для произвольного начального распределения. Оно содержит бесконечный набор констант $f_{i}(0)$. На практике обычно интересуются ситуацией с $f_{i}(0)=\delta_{i, 0}$ (монодисперсное начальное условие), которая является частным случаем распределения Пойа при $M_{1}(0)=0$. В следующем пункте будет показано, что для начального условия в виде распределения Пойа решение представимо в значительно более простой форме.

\section{4. КАНОНИЧЕСКАЯ ИНВАРИАНТНОСТЬ}

Рассмотрим частный случай решения (38) при

$$
f_{i}(0)=\left[1+\alpha M_{1}(0)\right]^{-1 / \alpha} \frac{\prod_{j=0}^{i-1}(1+j \alpha)}{i !}\left[\frac{M_{1}(0)}{1+\alpha M_{1}(0)}\right]^{i} .
$$

Подстановка (40) в (38) с учетом (34) и (19) дает

$$
f(z, t)=\left[1+\alpha M_{1}(t)(1-z)\right]^{-1 / \alpha}
$$

т.е. производящая функция сохраняет первоначальный вид при всех $t$. Следовательно, сохраняется во времени и начальное распределение Пойа:

$$
f_{i}(t)=\left[1+\alpha M_{1}(t)\right]^{-1 / \alpha} \frac{\prod_{j=0}^{i-1}(1+j \alpha)}{i !}\left[\frac{M_{1}(t)}{1+\alpha M_{1}(t)}\right]^{i} .
$$

Зависимость от времени теперь осушествляется только через средний размер, по-прежнему определяемый уравнением $(31)$ при $f_{A}(t)=\Gamma-M_{1}(t)$ для замкнутой системы 
или (33) для открытой системы. Это свойство позволяет говорить о канонической инвариантности рассматриваемой модели. Так, в [5, 6] было показано, что в случае колебательной релаксации газа гармонических осцилляторов наблюдается сохранение начального больцмановского распределения с изменяющейся колебательной температурой. Аналогично можно сказать, что система управляющих уравнений (3)-(5) с линейными по $i$ константами скоростей обладает свойством канонической инвариантности относительно распределения Пойа с меняюшимся средним размером комплексов.

Отметим некоторые особенности распределения (42). При $\alpha=1$ оно совпадает с геометрическим распределением

$$
f_{i}(t)=M_{1}^{i}(t) /\left(1+M_{1}(t)\right)^{i+1}
$$

которому можно придать больцмановский вид, если положить $M_{1}(t) /\left(1+M_{1}(t)\right) \equiv$ $\exp (-\epsilon(t))$. В пределе $\alpha \rightarrow 0$, соответствующем одинаковой для всех размеров константе скорости роста $k^{+}$, распределение (42) переходит в распределение Пуассона

$$
f_{i}(t)=\exp \left(-M_{1}(t)\right) M_{1}^{i}(t) / i !
$$

Распределение Пойа имеет дисперсию

$$
D(t)=M_{1}(t)\left(1+\alpha M_{1}(t)\right)
$$

т.е. разброс размеров комплексов возрастает с увеличением $\alpha$. Минимальный разброс соответствует распределению Пуассона.

\section{5. НЕКОТОРЫЕ ЧАСТНЫЕ СЛУЧАИ РЕШЕНИЙ}

Для материально изолированной системы из уравнений $(31),(10)$ получаем следующее уравнение на средний размер:

$$
d_{t} M_{1}(t)=-\alpha k^{+} M_{1}^{2}(t)+\left(\alpha k^{+} \Gamma-k^{+}-k^{-}\right) M_{1}(t)+k^{+} \Gamma .
$$

Решение (46) имеет вид $(\alpha>0)$

$$
\begin{aligned}
M_{1}(t) & =\frac{a_{1}+\left|a_{2}\right| \exp \left(-\alpha k^{+}\left(a_{1}+\left|a_{2}\right|\right) t\right)}{1-C \exp \left(-\alpha k^{+}\left(a_{1}+\left|a_{2}\right| t\right)\right)} \\
a_{1,2} & \equiv \frac{1}{2}\left(\Gamma-\frac{k^{+}+k^{-}}{\alpha k^{+}}\right) \pm \frac{1}{2}\left[\left(\Gamma-\frac{k^{+}+k^{-}}{\alpha k^{+}}\right)^{2}+\frac{4 \Gamma}{\alpha}\right]^{1 / 2}, \\
C & \equiv\left(M_{1}(0)-a_{1}\right) /\left(M_{1}(0)+\left|a_{2}\right|\right) .
\end{aligned}
$$

Функция $G(t)$, входящая в решение (38) для произвольных начальных распределений, получается из (47) при $C=-a_{1} /\left|a_{2}\right|$. При $t \rightarrow \infty \quad M_{1}(\infty)=a_{1}, f_{A}(\infty)=\Gamma-a_{1}$, что согласуется с (24). 
Рассмотрим подробнее случай $\alpha \rightarrow 0$. При пуассоновских начальных распределениях решение дается выражением (44). В случае произвольных $f_{i}(0)$ производящая функция (38) при $\alpha=0$ принимает более простой вид

$$
\begin{aligned}
f(z, t) & =\exp [G(t)(z-1)] \sum_{i=0}^{\infty} f_{i}(0)[1+b(t)(z-1)]^{i}, \\
b(t) & =\exp \left(-k^{-} t\right) ; \quad G(t)=M_{1}(t)-M_{1}(0) b(t) .
\end{aligned}
$$

Восстанавливая функцию распределения из (48), (49), получаем

$$
f_{i}(t)=\left.\exp (-G(t)) \frac{[G(t)+b(t) \partial z]^{i}}{i !} f(z, 0)\right|_{z=1-b(t)} .
$$

Следовательно, концентрации $f_{i}(t)$ получаются применением пуассоновского дифференциального оператора к производяшей функции при $t=0$ с последуюшей заменой $z$ на $1-b(t)$

В отсутствие распада комплексов $\left(k^{-}=0\right)$ выражение (50) сводится к известному результату [3]

$$
f_{i}(t)=\sum_{j \geqslant 0} T_{i j}(t) f_{j}(0)
$$

где пропагатор $T_{i j}(t)$ имеет вид

$$
\begin{gathered}
T_{i j}(t)=\exp (-G(t)) G^{i-j}(t) /(i-j) !, \quad 0 \leqslant j \leqslant i, \\
T_{i j}(t)=0, \quad j>i, \\
G(t)=M_{1}(t)-M_{1}(0)=k^{+} \int_{0}^{t} d t^{\prime} f_{A}\left(t^{\prime}\right) .
\end{gathered}
$$

Разумеется, в случае $k^{-}=0 \quad M_{1}(t) \rightarrow \infty$ при $t \rightarrow \infty$.

Уравнение (32) для среднего размера при $\alpha=0$ становится линейным и может быть исследовано для широкого класса источников. В простейшем случае $P(t)=P=$ const и $Q(t)=Q=$ const решение $(32),(37)$ есть

$$
\begin{gathered}
f_{A}(t)=P / Q+C_{1} \exp \left(-\lambda_{1} t\right)+C_{2} \exp \left(-\lambda_{2} t\right), \\
M_{1}(t)=k^{+} P / k^{-} Q+\left(\lambda_{2} / k^{-}-1\right) C_{1} \exp \left(-\lambda_{1} t\right)+\left(\lambda_{1} / k^{-}-1\right) C_{2} \exp \left(-\lambda_{2} t\right), \\
\lambda_{1,2} \equiv \frac{1}{2}\left(k^{+}+k^{-}+Q\right) \mp \frac{1}{2}\left[\left(k^{+}+k^{-}+Q\right)^{2}-4 k^{-} Q\right]^{1 / 2} \\
C_{1} \equiv f_{A}(0)-\frac{\lambda_{2}\left(f_{A}(0)+P / Q\right)-P-k^{-} \Gamma(0)}{\lambda_{2}-\lambda_{1}} \\
C_{2} \equiv \frac{\lambda_{2} f_{A}(0)+\lambda_{1} P / Q-P-k^{-} \Gamma(0)}{\lambda_{2}-\lambda_{1}}
\end{gathered}
$$

Очевидно, $\lambda_{2}>\lambda_{1}>0$, поэтому $f_{A}(\infty)=P / Q$ и $M_{1}(\infty)=k^{+} P / k^{-} Q$, что совпадает c (14), (22) при $\alpha=0$. Заметим, что в данном случае неравенство (23) заведомо выполнено, поэтому равновесное состояние с $M_{1}(\infty)=k^{+} P(\infty) / k^{-} Q(\infty)$ достигается при любых источниках $P(t), Q(t)$. 
Для материально изолированной системы при $\alpha=0$ вместо (47) имеем выражения

$$
\begin{aligned}
M_{1}(t) & =C^{+} \Gamma-\left(f_{A}(0)-C^{-} \Gamma\right) \exp \left[-\left(k^{+}+k^{-}\right) t\right], \\
f_{A}(t) & =C^{-} \Gamma-\left(f_{A}(0)-C^{-} \Gamma\right) \exp \left[-\left(k^{+}+k^{-}\right) t\right],
\end{aligned}
$$

где $C^{ \pm} \equiv k^{ \pm} /\left(k^{+}+k^{-}\right)-$приведенные значения констант скоростей реакции $A_{1}+$ $B \rightleftharpoons A_{1} B$.

\section{ЗАКЛЮЧЕНИЕ}

Таким образом, для линейных по числу частиц констант скоростей роста и распада удается получить точное решение системы управляюших уравнений. Это решение можно обобщить на случай переменного числа ядер, а также наличия источников комплексов различного размера. Возможно, точное решение может быть построено и для квадратичной зависимости $k_{i}^{+}=k^{+}\left(1+\alpha i+\beta i^{2}\right), k_{i}^{-}=k^{-}\left(i+\gamma i^{2}\right)$. В этом случае для производяшей функции будем иметь уравнение в частных производных второго порядка. Представляет интерес исследование нелинейного уравнения для среднего размера при $\alpha>0$, режимов с мошной накачкой, обобшение модели на случай учета межкластерных парных реакций. Указанные вопросы, а также возможности применения полученных результатов к некоторым конкретным задачам будут рассмотрены в дальнейшем.

\section{Список литературы}

[1] Ф. М Куни, Т. Ю. Новожилова, И. А. Терентьев // ТМФ. 1986. Т. 60. № 2. С. 276.

[2] А.В. Богданов, Ю.Е. Горбачев, Г. В. Дубровский, А. Л. Иткин, Е. Г. Колесниченко, В. М. Стрельченя // Химическая физика. 1989. Т. 8. №5. С. 677.

[3] J. B. Adams, W. N. G. Hitchon // J. Comput. Phys. 1988. V. 76. № 1. P. 159.

[4] Б. Ф. Гордиеи, А.И. Осипов, Л. А. Шелепин. Кинетические процессы в газах и молекулярных лазерах. М.: Наука, 1980.

[5] E. W. Montroll, K. E. Shuler // J. Chem. Phys. 1957. V. 26. №5. P. 454.

[6] C. C. Rankin, J. C. Light // J. Chem. Phys. 1967. V. 46. №4. P. 1305.

[7] А. П. Васильев, Г. В. Дубровский, В. М. Стрельченя // ПМТФ. 1984. №5. С. 22.

[8] J. Q. Searcy // J. Chem. Phys. 1974. V. 61. № 12. P. 5282.

[9] М. Эрнст // Фракталы в физике / Ред. Л.Пьетронеро и Э.Тозатти. М.: Мир, 1988.

[10] А. А. Лушников, А. Г. Сутугин // Усп. химии. 1976. Т. 45. № 7. С. 386.

[11] В. Г. Дубровский // Коллоидн. журн. 1990. Т. 52. № 2. С. 243.

[12] B. Caroli, C. Caroli, B. Roulet // J. Stat. Phys. 1979. V. 21. P. 415.

[13] А. М. Головин // Изв. АН ССР, сер. Геофизика. 1963. Вып. 5. С. 783.

[14] С. И. Кучанов // ДАН СССР. 1976. Т. 229. С. 135.

[15] R. J. Glauber // J. Math. Phys. 1963. V. 4. №3. P. 294.

[16] Ф. М. Куни, А. П. Гринин // Вестн. ЛГУ. 1982. №22. С. 10.

Институт аналитического приборостроения РАН Санкт-Петербург

\section{V.G. Dubrovsky \\ ON THE EXACT SOLUTION OF MASTER EQUATIONS OF REVERSIBLE GROWTH MODEL}

A set of master equations of reversible quasichemical growth model in a hetorogeneous materialy open system is studied. For the case of size-linear rate constants, the exact analytical solution of master equations is obtained. It is shown that the model is canonically invariant with respect to the Polya size distribution with a variable mean size of clusters. 